\title{
Mass Loss Processes in Hot Evolved Stars: Planetary Nebula Nuclei in the Large Magellanic Cloud
}

\author{
L. Bianchi $^{1,2}$, E. Vassiliadis ${ }^{3}$ and M.A. Dopita ${ }^{4}$ \\ ${ }^{1}$ Johns Hopkins University, USA; ${ }^{2}$ Osservatorio Astronomico di Torino, Italy; \\ ${ }^{3}$ Instituto de Astrofísica de Canarias, Spain; \\ ${ }^{4}$ Mount Stromlo and Siding Spring Observatories, Australia
}

As part of a broad study of stellar winds in different galaxies, to establish observational constraints on the influence of metallicity on the mass loss process, we investigate the winds of planetary nebula (PN) nuclei in the Large Magellanic Cloud. Theoretically, the radiation pressure mechanism implies a strong dependence of the wind acceleration on the element abundances. Observationally, model predictions have been tested using Pop. I stars in the Magellanic Clouds (Puls et al. 1996, A\&A, 305, 171) and massive stars in the nearby galaxies M31, M33 and NGC 6822 (Bianchi et al. 1996, AJ, 111, 2303; Bianchi et al. 1996, in prep.). We now extend this investigation to low luminosity, evolved objects.

We analyze the UV P-Cygni profiles of three PN nuclei in the Large Magellanic Cloud (LMC) using the SEI method (Lamers et al. 1987, ApJ, 314, 726). The data are taken with the Hubble Space Telescope Faint Object Spectrograph (Vassiliadis et al. 1996, ApJS, $105,375)$. The wind velocities are comparable to those found in Galactic PN nuclei of similar effective temperature, but a definitive comparison must await the determination of the spectral types and masses of the central stars. The continuum distribution (1150-7800 $\AA$ ) is modeled to isolate the stellar and nebular contributions. For one object of WR-type, LMC-SMP61, we derive an upper limit to the mass loss rate of $7 \times 10^{-7} \mathrm{M}_{\odot} \mathrm{yr}^{-1}$. 